\title{
CUATRO IDEAS SOBRE LA VIDA DESDE EL HORIZONTE DE LA TÉCNICA*
}

\author{
FOUR IDEAS ABOUT LIFE FROM THE HORIZON \\ OF THE TECHNIQUE
}

\section{ESTEBAN VARGAS**}

\section{RESUMEN}

En este trabajo intentamos mostrar cómo cuatro grandes pensadores (Aristóteles, Descartes, Lamarck y Darwin) han estudiado la vida desde el horizonte de la técnica de su época. Aristóteles entiende la vida desde la técnica griega que crea objetos que imitan la naturaleza; Descartes desde los “autómatas" como el reloj; Lamarck, desde la herencia cultural y, finalmente, Darwin, desde la selección artificial del hombre. Este artículo presenta algunos textos de estos autores para apoyar estas ideas y plantea algunas interrogantes al final de la conclusión.

Palabras clave: Aristóteles, Descartes, Lamarck, Darwin, vida, técnica.

\section{ABSTRACT}

In this paper we try to show how four great thinkers (Aristotle, Descartes, Lamarck, and Darwin) studied life from the horizon of the technique of their time. Aristotle understands life from the Greek technique that creates objects that mimic nature; Descartes from the "automata" as the clock; Lamarck, from the cultural heritage; and finally, Darwin, from the artificial selection of man. This article presents some texts by these authors to support these ideas and raises some questions at the end of the conclusion.

Keywords: Aristotle, Descartes, Lamarck, Darwin, life, technique.

Recibido: 01.06.17. Aceptado: 15.08.17.

* Este artículo fue posible gracias a un Proyecto Fondecyt Regular Nº 1140922 llamado "Ciencia y realidad en Zubiri".

** Doctor en Filosofía. Profesor de la Pontificia Universidad Católica de Valparaíso. Valparaíso, Chile. Correo: esteban.vargas@pucv.cl. 


\section{INTRODUCCIÓN}

S

UELE DECIRSE que la ciencia se caracteriza principalmente por estudiar la "realidad objetiva", el hecho puro y objetivo que se encuentra "ahí delante" para posteriormente, a partir de ese "dato", poder elaborar una hipótesis y un cuerpo de explicaciones. No hay en esta idea ninguna influencia del quehacer humano o bien, a lo sumo, se acepta que esa influencia afecta sólo al momento psicológico de elaboración de hipótesis y teorías. Sin embargo, si miramos de cerca la historia del pensamiento científico, vemos la notable influencia del medio cultural que ha habido en los científicos. La ciencia no se ha hecho a espaldas de su época. Esto puede apreciarse, por ejemplo, en la historia del pensamiento biológico. En este escrito se pretende mostrar brevemente algunos hitos cruciales del pensamiento biológico en relación a su concepción de vida y su relación con la técnica de cada época. Esto lo hacemos escogiendo cuatro pensadores teóricos de la vida lo suficientemente importantes como para incluirlos en esta exposición.

Antes de iniciar la exposición convendría señalar brevemente qué se entiende aquí por técnica y por horizonte. Técnica, considerada en tercera persona ${ }^{1}$, es ante todo un saber productivo, un "hacer" del hombre que consiste en saber producir o crear cosas. La carpintería, la ingeniería, etc., son, en este sentido, saberes técnicos, pues producen algo como un mueble, un edificio, etc. ${ }^{2}$. Pero "técnica" puede significar algo más: ya no es algo que el hombre "hace" sino un "modo de ser" de las cosas ante el hombre. Es una concepción más bien "en primera persona” de la técnica, que ha meditado, por ejemplo, Heidegger".

Ahora bien, en este escrito intentamos seguir ambos sentidos de la palabra "técnica" indiscernidamente. Lo importante aquí no es explicar qué es la técnica, sino considerarla (sea lo que ella fuere) como "horizonte" de ex-

\footnotetext{
${ }^{1}$ Por "tercera persona" se entiende aquí el estudio de lo que el hombre hace, lo que podemos describir observando a los demás. Este sentido de la "técnica” lo explica Ortega y Gasset así: "Si se considera al hombre -desde afuera-como el otro, el mejor método es el behaviorista, la investigación del comportamiento [...]. Entre los movimientos del otro hombre, que podemos observar, hay un grupo muy interesante: los movimientos técnicos. Se trata de los manejos que realiza el hombre cuando fabrica un objeto" (2014, p. 174).

${ }^{2}$ Carl Mitcham, en este sentido, define la técnica como el "conjunto de procedimientos puestos en práctica para obtener un resultado determinado. Existe la técnica de la caza, de la pesca, de la danza, de cocinar, de contar cuentos" (1989, pp. 13-14).

${ }^{3}$ Así Heidegger nos dice: "La técnica no es pues un mero medio, la técnica es un modo del salir de lo oculto. Si prestamos atención a esto se nos abrirá una región totalmente distinta para la esencia de la técnica. Es la región del desocultamiento, es decir, de la verdad” (2001, p. 14).
} 
plicación, en este caso, de lo que es un ser vivo. ¿Qué es horizonte? Siguiendo a Zubiri, entendemos aquí por horizonte el "medio" en que se intelige algo. A este "horizonte" Zubiri lo llama más precisamente "campo". En toda intelección se nos actualiza algo real desde la "unidad" de las demás cosas reales (campo). Campo no es una cosa real más que se intelige, sino aquello "desde" lo cual inteligimos algo. Es un "medio" de intelección, algo que nos "hace ver" las cosas de una determinada manera (Zubiri, 1982, p. 76). Es el medio como "luz" o ser de las cosas.

En este escrito sostenemos que, en muchos casos, la intelección de qué es un ser vivo se ha construido a la "luz" del campo u horizonte técnico de cada época, es decir, en contraposición de un determinado objeto o aspecto técnico. Con esto no queremos sostener que este horizonte ha sido decisivo en la "historia interna" del quehacer biológico. Lo único que queremos mostrar es que muchas concepciones de la vida se han construido dentro del horizonte técnico que les ha tocado en suerte.

\section{1) ARISTÓTELES}

El primer pensador que vislumbró la relación entre ser vivo y técnica, y que, de algún modo, marcó el pensamiento de occidente sobre este punto fue Aristóteles. Este pensador intentó comprender los seres vivos como seres naturales desde su teoría de la causalidad, la cual es iluminada desde el horizonte de la técnica griega. Veamos esto en tres pasos.

a) Naturaleza y técnica. Para Aristóteles, naturaleza y técnica son principios de las cosas. ¿Qué es principio? Aristóteles dice que principio es "lo primero a partir de lo cual algo es, o se produce, o se conoce" (2000, p. 195). Aristóteles parece entender naturaleza y técnica como principios de "producción" de las cosas, el segundo de los tres sentidos mencionados en la cita. La naturaleza es un principio de producción ex se, desde sí mismo; la técnica, en cambio, es un principio "por otro", es producto de un técnico o artesano (Aristóteles, 2000, p. 462). Así, para saber si una entidad es natural o técnica (artificial), hay que preguntarse cómo se ha producido o generado. Por ejemplo, una silla es una entidad técnica, producida por un técnico, no así sus materiales que son naturales.

Pero naturaleza y técnica podrían entenderse también en el primer sentido de principio de Aristóteles, es decir, aquello primero a partir de lo cual algo "es". Es el punto de vista en que, en cierto modo, se ha colocado Heide-

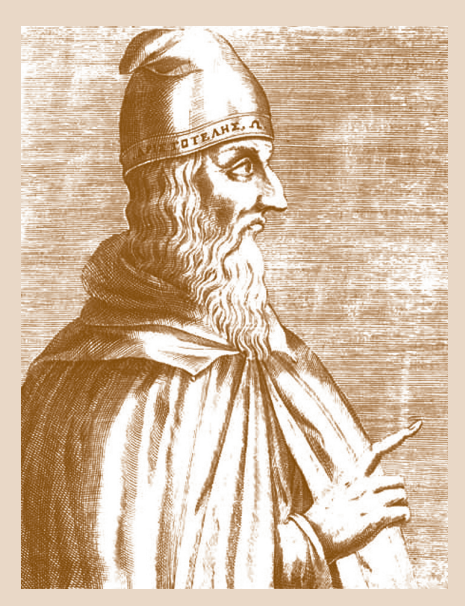

Aristóteles 
gger y Zubiri. Aunque no es nuestro tema, conviene aclararlo para perfilar mejor el escrito. Conservando la idea del principio por sí mismo y por otro (natural y técnico), podría replantearse lo que es natural y técnico en este primer sentido de principio. Para aclarar esto, tomemos, como ejemplos, una manzana en una obra de arte y un tomate transgénico. En el sentido de "principio de producción", la manzana es un ente natural, pero, colocada en una obra de arte, en un museo, su "modo de ser" ha cambiado. Su "ser artístico" se entiende ahora desde la mirada del artista o del público, no por sus meras propiedades intrínsecas. En el caso inverso está el tomate transgénico. Su producción, en cierta forma, se debe al hombre, y, en este sentido, es una entidad técnica, pero si lo miramos desde el primer sentido de principio es un ente natural en tanto en cuanto su "ser" se debe a sus propiedades intrínsecas. Si no se dice, las personas no saben si están comiendo un tomate transgénico. En este sentido, para saber si algo es natural o técnico ya no hay que preguntarse cómo ha sido producido sino de dónde arranca el principio de su modo de ser.

En este escrito tratamos de entender lo que "es" un ser vivo a la luz de la técnica de cada época en cuatro grandes pensadores. Un aspecto de la técnica ha servido de luz para entender lo que "son" los seres vivos. Volvamos ahora a la concepción de vida en Aristóteles

b) Los seres vivos como entidades naturales. Aristóteles intenta comprender los seres vivos como entidades "naturales" que se explican por sí mismos a partir de su teoría de las cuatro causas (material, formal, eficiente y final). Para efectos de nuestro tema, estas causas pueden reducirse a dos. En primer lugar, la causa "material", es decir, aquello de lo que están hechos los seres vivos. Según Aristóteles, éste es el punto de vista predominante en los filósofos anteriores a él como Empédocles. Desde este aspecto "material", los elementos que constituyen a los seres vivos se explican por propiedades "necesarias", como, por ejemplo, el elemento fuego que tiene "necesariamente" que subir, el elemento tierra bajar, etc. En el plano biológico, por ejemplo, la dureza de un diente se debe "necesariamente" a la constitución de los elementos que lo constituyen. Esto lo asume Aristóteles, pero le parece insuficiente, especialmente en el caso de los seres vivos. Esto porque éstos

\footnotetext{
${ }^{4}$ Por lo mismo, Zubiri entiende que nuestra técnica ya no puede ser entendida como un mero principio de producción por otro. Así lo señala: "Tal vez la técnica griega, y en general toda la técnica antigua, sólo pudiera realizar 'arte-factos', esto es, cosas que la naturaleza no produce y que una vez producidas no tienen 'actividad natural' [... Pero en nuestro mundo esto no es verdad (...) Nuestra técnica produce artificialmente entes naturales" (2008, pp. 84-85).
} 
son más que la sumatoria de los elementos materiales que los constituyen. Hay una cierta "organización" de esos elementos que no es reducible a las propiedades de sus partes. Esta "organización" se explica por la causa que podríamos llamar aquí "formal-final". Es causa "formal" en tanto le da una cierta "organización" a los elementos materiales, y causa "final" en tanto esa organización no es estática ni desordenada sino "dirigida" dinámicamente, es decir, es un proceso ordenado y secuenciado que "tiende" hacia algo "siempre o la mayoría de las veces" (por ejemplo, el desarrollo de un huevo de gallina conduce casi siempre al nacimiento del polluelo en una secuencia ordenada de etapas). En el caso de los seres vivos, esta "forma-fin" es el alma. El alma es el principio de que el viviente se automueva y esté organizado.

Ahora bien, la tesis contraria, la que Aristóteles piensa que defienden los materialistas griegos, parecería explicar los seres vivos sólo como una mera "adición" de elementos materiales por "casualidad" o azar. En la segunda de sus lecciones sobre Física (1995, pp. 162-163), Aristóteles nos muestra que en la tesis "materialista" de los seres vivos, cada una de las partes materiales de un ser vivo surgiría por separado y su "unión" sería por pura "casualidad", es decir, algo que no está necesariamente determinado. Por ello, una manera de explicar tal "unidad" de los seres vivos sería "consecutiva" a las partes: surgirían muchas partes que se unirían entre sí por azar o casualidad. Aquellas que por "casualidad" quedaron bien constituidas se mantendrían, el resto perecerían. Tal sería, según Aristóteles, la tesis de Empédocles. Es una tesis que es semejante a la de Darwin, como veremos más abajo. Ahora bien, esta explicación material, aunque necesaria, es insuficiente para Aristóteles. Ello porque en los entes naturales, como los seres vivos, su organización no es casual, ocurre "siempre o en la mayoría de los casos". Cuando se origina un hombre, éste en la mayoría de las veces posee dos ojos, dos orejas, etc. Si fuera por mera casualidad, esto ocurriría rara vez, lo cual no se observa. Es importante destacar que la finalidad de un ser vivo en Aristóteles no se prueba aquí porque alguien ha "planeado" a un ser vivo para que sea así, sino porque su dinámica "tiende" siempre o la mayoría de las veces a un mismo resultado.

c) Los seres vivos y la técnica griega. Aristóteles trata de entender las entidades naturales como los seres vivos en "analogía" con los entes técnicos creados por el hombre como, por ejemplo, una casa ${ }^{5}$. Así, Aristóteles sostiene

\footnotetext{
5 "Por ejemplo, si una casa hubiese sido generada por la naturaleza, habría sido generada tal como lo está ahora por el arte [técnica]. Y si las cosas por naturaleza fuesen generadas no sólo por
} 
que el proceder técnico es semejante, pero no idéntico, al proceder natural. De hecho, se sostiene que es la técnica la que imita o completa la naturaleza. Esta analogía se basa en cuatro momentos esenciales:

$\left.1^{\circ}\right)$ Aristóteles intenta entender a los seres vivos en función de los artefactos producidos por la técnica griega. Tales artefactos se caracterizan, al menos, por dos propiedades. Por un lado, los objetos producidos, como dice Aristóteles, "completan o imitan a la naturaleza" (el bastón al pie, la sierra a los dientes, etc.). Por otro lado, tales objetos, en general, no poseen actividad "por sí mismos", algo propio, como veremos, de la técnica moderna.

$2^{\circ}$ ) En toda elaboración técnica hay aquello que debe ser forjado, a saber, la "materia" entendida como el "material". Por ejemplo, en el caso de la casa serían sus maderas, sus piedras, etc. Lo mismo sucedería, por ejemplo, en los seres vivos. Pero ésta es claramente una concepción "técnica" de la materia. La materia es entendida aquí como lo meramente "potencial", como aquello que, por decirlo así, está esperando el poder ser ordenada. La conexión, por ejemplo, de una piedra con otra es puramente "extrínseca" donde justamente se requiere de un principio ordenador (el técnico). Pero ipodría decirse hoy que las biomoléculas, células, tejidos, etc., de un organismo son el "material" de un ser vivo en la misma forma que lo es una piedra en una casa?

30) En ese quehacer técnico también hay un principio organizador que "ordena" la materia potencial. Es la "forma". Claro está que Aristóteles ve una gran diferencia entre la procedencia de la forma técnica con la forma natural. En el quehacer técnico, este principio viene dado por el técnico, en cambio, en los seres vivos es un principio natural intrínseco que llama "alma". No obstante ello, la forma sigue concibiéndose en forma "técnica" como un principio "ordenador". $\mathrm{Y}$ esto es algo que habría que discutir en la biología actual: ¿hay un principio "ordenador" de la realidad material, por ejemplo, del cerebro?

la naturaleza sino también por el arte [técnica], serían generadas tales como lo están ahora por la naturaleza. Así, cada una espera la otra. En general, en algunos casos el arte [técnica] completa lo que la naturaleza no puede llevar a término, en otros imita a la naturaleza" (Aristóteles, 1995, p. 164-165). 
$\left.4^{\circ}\right)$ Por último, y lo que más se ha resaltado, en el quehacer técnico hay una cierta finalidad, un cierto "para qué". Lo mismo destaca Aristóteles en los seres vivos, aunque, como vimos, con la salvedad de que ese "fin" no tiene que resultar necesariamente de un "proyecto", propio de la finalidad técnica. Como quiera que sea, la finalidad en los seres vivos es meditada en analogía al quehacer técnico como una ordenación final.

Resumiendo, Aristóteles ha colocado como ejemplo los casos propios del quehacer técnico griego para entender mejor la naturaleza de los seres vivos. Por ello dice que "la técnica imita la naturaleza". Pero podría pensarse perfectamente al revés, es decir, que ha sido una determinada concepción del quehacer técnico griego la que ha servido para "iluminar" la naturaleza del ser vivo. Tomando el proceso técnico que tiene a la mano (hacer casas, etc.), lo usa como modelo (mutatis mutandi) para entender el proceso de los seres vivos en su aspecto material, formal y final. En este caso, bien se podría decir, al revés, que, en cierto modo, "la (concepción de) naturaleza (biológica) es la que imita a la técnica".

\section{2) DESCARTES}

Bastantes siglos más tarde hace su entrada otro gran pensador de lo vivo. Sabido es que Descartes ha distinguido dos grandes tipos de realidad; la realidad pensante y la realidad extensa o material. La primera se da en el hombre y la segunda constituye a todos los entes físicos, incluido un cierto aspecto del hombre mismo. Ahora bien, desde esta concepción, Descartes pretende entender a los seres vivos a quienes concibe como realidades extensas organizadas. Para entender esto, tengamos en cuenta que una realidad extensa consiste esencialmente "en ser una cosa extendida en largo, ancho y profundidad" (Descartes, 1951, p. 43). Lo "extenso" posee una serie de momentos orientados los unos a los otros. De esta concepción ha surgido justamente la geometría analítica que el mismo Descartes ha desarrollado. A partir de esta concepción, Descartes intenta comprender a los seres vivos. Los entiende como "cuerpos", es decir, como una serie de elementos ordenados intrínsecamente los unos a los otros. Todas las propiedades de un ser vivo se pueden explicar en la "funcionalidad" de unos elementos a otros. Así, por ejemplo, la locomoción puede ser entendida como un proceso funcional de contracción muscular posibilitado por los nervios que contienen

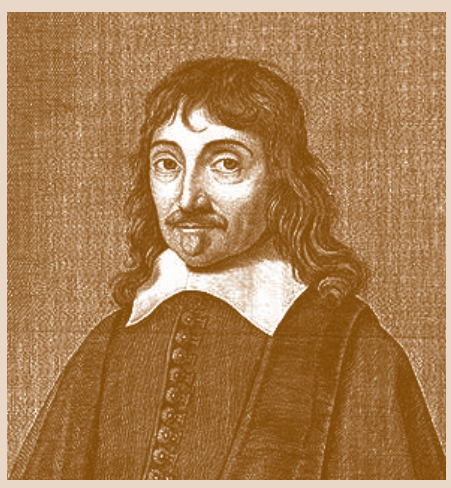

R. Descartes 
un aire sutil llamado por Descartes "espíritu animal”. Más allá de esta explicación, este pensamiento abrió las puertas a lo que se ha llamado el "fisicismo" o mecanicismo biológico del cual ha nacido la moderna fisiología.

Pero esta explicación también está en abierta relación con la técnica de su tiempo. Así, según Las pasiones del alma ${ }^{6}$, Descartes entiende que el cuerpo, con sus diversos elementos ordenados, puede moverse por sí mismo como, por ejemplo, en los reflejos inconscientes. Pero esto mismo también ocurre en un autómata como el reloj. Y, dado que el reloj no tiene alma y se automueve, ahora no se requiere pensar el alma como principio del movimiento corporal o material ${ }^{7}$. De ahí entonces que Descartes diga que la muerte no es ausencia del alma, sino que ocurre por disfunción o corrupción al modo como se estropea un reloj. La materia tiene ahora en Descartes una cierta ordenación de por sí.

En segundo lugar, destaquemos más explícitamente la conexión que hace Descartes con las máquinas. Las máquinas de esta época tienen automovimiento como el reloj, cosa que es muy poco usual en el mundo griego. Poseen una actividad propia. Si antes Aristóteles trata de entender al ser vivo desde los artefactos que imitan o completan la naturaleza (como la sierra), ahora Descartes lo trata de entender desde los autómatas como el reloj y desde aquel horizonte técnico que ha permitido forjarlos. El hombre con su "pensar analítico" ha podido forjar autómatas como el reloj y con la misma mirada ha tratado de entender a los seres vivos. Dado que las partes de un reloj tienen una cierta coherencia interna, Descartes ha entendido las partes de un organismo del mismo modo. Ahora Descartes no requiere del alma como un principio inmaterial ordenador del movimiento material, por ejemplo, para explicar un reflejo. El reflejo rotuliano puede explicarse perfectamente desde la funcionalidad interna de sus elementos componentes como un "reflejo autónomo". Ello, como decíamos, trajo grandes posibilidades para la biología, especialmente para los procesos fisiológicos. Pero ese mismo pensamiento dejó en la oscuridad el carácter evolutivo de

\footnotetext{
6 "Consideremos que la muerte no ocurre nunca por ausencia del alma, sino solamente porque alguna de las principales partes del cuerpo se corrompe; y pensemos que el cuerpo de un hombre vivo difiere del de un hombre muerto lo mismo que un reloj u otro autómata (es decir, cualquier otra máquina que se mueva por sí misma), cuando está montado y tiene en sí el principio corporal de los movimientos para los cuales fue creado, con todo lo necesario para su funcionamiento, difiere del mismo reloj o de otra máquina cuando se ha roto y deja de actuar el principio de su movimiento" (Descartes, 2005, p. 86).

${ }^{7}$ Descartes entiende el alma como aquello inmaterial que tiene conciencia. A diferencia de Aristóteles, para Descartes las plantas y animales no tienen alma o psique, sino sólo el hombre.
} 
los seres vivos. ¡Cuándo se ha visto que un reloj evolucione por sí solo en tanto reloj! Esto es lo que intentarán explicar los dos próximos pensadores.

\section{3) LAMARCK}

Este pensador tuvo su apogeo a comienzos del siglo XIX. Es famoso en biología por dos aportes. En primer lugar, por intentar una nueva clasificación de los seres vivos introduciendo, por ejemplo, la distinción entre animales vertebrados e invertebrados. Para hacer esta clasificación, Lamarck intenta diferenciar el orden natural o filosófico (dado por sí mismo) y el orden técnico o económico dado por el hombre con un fin meramente de utilidad. De este modo, las distinciones entre taxones como clases, órdenes, etc., y la nomenclatura de los seres vivos no son puramente naturales, sino que obedecen a un criterio técnico de utilidad (Lamarck, 1986, p. 38). No obstante ello, Lamarck piensa que puede encontrarse un cierto orden en la naturaleza siguiendo la conexión natural entre unos organismos y otros. Esta conexión le muestra a Lamarck un orden jerárquico ordenado naturalmente desde el organismo más simple (los infusorios o células) hasta el más complejo (el hombre). En este sentido, Lamarck piensa la naturaleza como los antiguos, es decir, como un principio en y por sí mismo, independiente de la técnica humana, pero, como él diría, en un orden inverso de generación, desde lo más simple a lo más complejo. Empieza ya a cuestionarse ese viejo principio metafísico del "nadie da lo que no tiene", que implicaba que lo más complejo no podría provenir de lo más simple.

En segundo lugar, su otro gran aporte es postular una teoría evolutiva. Lamarck sostiene que hay una tendencia natural hacia una mayor complejidad, tendencia explicada básicamente por dos leyes (1986, p. 175). Su primera ley nos muestra que el frecuente uso de un órgano, por ejemplo, de un músculo, lo desarrolla, mientras que su desuso lo atrofia. Su segunda ley es la teoría de la herencia de los caracteres adquiridos, sujeta a múltiples críticas. En ella nos dice que un órgano que un individuo use en demasía lo desarrollará y tal desarrollo pasará a la descendencia. Así, él explicaría, por ejemplo, la evolución de la jirafa, la que para alcanzar los árboles más grandes estira el cuello, pasando este desarrollo a la descendencia, por lo cual sus hijos nacerán con el cuello un poco más largo que sus padres. Por el contrario, si un individuo no usa un órgano, éste se irá atrofiando poco a poco y esto pasará a la descendencia. Así explicaría, por ejemplo, el caso del topo, quien tiene ojos atrofiados, los cuales se han ido perdiendo paulati-

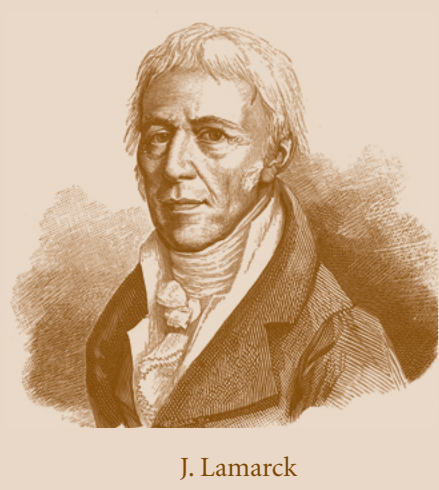

J. Lamarck 
namente debido al poco uso de ellos, por vivir este animal en la oscuridad. De esta forma los organismos, según sus posibilidades, pueden irse poco a poco adaptando mejor a las condiciones del medio. Es justo esta explicación la base para entender la idea de "progreso".

Estas leyes le parecen a Lamarck indudables. El pensamiento clásico, según Lamarck, pensaba que las estructuras o formas determinan a priori sus posibles usos o funciones. De ahí que el accionar de una forma no puede hacer variar a la misma forma. Por ello no podía haber evolución y las formas eran eternas. Pero ahora nos invita a pensar lo contrario ${ }^{8}$. Ahora bien, aquí hay que pensar si esta idea surge de la "mera observación", como dice Lamarck, o si hay alguna influencia técnico-cultural. Un poco más bajo, Lamarck nos dice: "Hace ya mucho tiempo que se ha tenido a este respecto el sentimiento de la verdad. Nos lo prueba el proverbio que todo el mundo conoce: Los hábitos forman una segunda naturaleza. Ciertamente si los hábitos y la naturaleza de cada animal no pudiesen cambiar nunca, el proverbio habría resultado falso, lo cual no sucede" (1986, p. 176). El proverbio, así como Lamarck lo entiende, no viene inspirado del decir popular, sino, en definitiva, de las ideas de Aristóteles. Un hábito es una disposición adquirida con el uso reiterado, como aprender a tocar el piano. Estos hábitos son una especie de "naturaleza" porque, ciertamente, una vez adquiridos, nos "brotan" de modo natural (como el fluir de los dedos de un pianista en un concierto), pero son naturaleza "segunda", precisamente porque no se traen al nacer ni se heredan a la descendencia. Lo que Lamarck sostiene es que estos hábitos pueden heredarse a la descendencia, con lo cual de "segunda" naturaleza (o adquiridos) pasan a ser de "primera" naturaleza o constitutivos. De este modo, entonces, la distinción entre primera y segunda naturaleza, desde esta concepción, sería sólo temporal.

Supuesto todo esto, debo señalar que no me parece que éste sea el sentido radical que todo el mundo reconoce en el proverbio. La experiencia que nos es más familiar del adquirir, heredar y progresar es lo que ocurre en lo que se suele llamar la "herencia cultural". Pensemos, por ejemplo, en la historia de la plancha. Este artefacto es creado para resolver la necesidad de alisar la ropa con el mínimo esfuerzo y gasto energético. Es la misma necesidad la que exige mejoras que se van produciendo paulatinamente.

${ }^{8}$ Así nos dice que "es fácil demostrar por la observación que son, por el contrario, las necesidades y los usos de las partes los que las desarrollaron [las formas], y hasta los han hecho nacer cuando no existían, dando lugar al estado en que las vemos en cada animal" (Lamarck, 1986, p. 176). 
Sólo supuesta esta necesidad "invariable" es desde donde se puede decir que en las planchas hay un "progreso": ellas se fabrican cada vez más eficientes para su originaria utilidad. Ahora bien, desde este horizonte es desde donde queda iluminada la idea de evolución de Lamarck. En el lenguaje coloquial, evolución es sinónimo de progreso. Lamarck pensó los seres vivos, al igual que Descartes, como máquinas. De hecho intentó explicarse la influencia mecánica que tendría la voluntad y el uso sobre el desarrollo y hasta en la creación de los órganos. Pero pensó algo más, pensó que los "seres vivosmáquinas" tienen herencia con modificación, cosa que no le sucede al reloj de Descartes ni a su concepción de ser vivo. Sin embargo, la herencia es pensada aquí, a mi juicio, en la "familiaridad" de la herencia cultural en la cual el hombre genera ideas que transmite a los demás, que genera artefactos y los perfecciona, etc. De hecho, cuando hoy se intenta enseñar biología evolutiva, la gente la entiende rápidamente al estilo Lamarck. Así, se piensa que por desuso se perderán las muelas del juicio, etc., como se piensa que por uso el cráneo seguirá creciendo, etc. La herencia cultural nos es sumamente familiar, puesto que pertenece a nuestro "horizonte técnico", pero no así la idea de una herencia biológica de caracteres adquiridos. De hecho, la biología, en general, no ha podido demostrar que ocurra esto último.

\section{4) DARWIN}

Finalmente, tratemos de entender el pensamiento de Darwin. Este pensador también trató de entender la evolución de los seres vivos y, aunque asumió algunos supuestos del pensamiento de Lamarck, aportó indudablemente nuevas ideas al respecto. ¿Cuál es el aporte de Darwin? Podemos resumir básicamente el pensamiento de Darwin en dos momentos o principios fundamentales. Por un lado, la constatación de la existencia de "variación" entre los individuos de una misma especie que habitan en un lugar y tiempo determinado. Es lo que hoy llamaríamos una variación poblacional. Esta variación, para Darwin, es "azarosa", no en el sentido en que no tenga causas, sino en el sentido en que es ciega, no está dirigida hacia la adaptación. Esta idea es semejante a la idea de Empédocles, según Aristóteles, como hemos visto. Por otro lado, está el hecho de la "competencia", la cual se produce dado que nace un mayor número de individuos de los que el medio puede soportar (por falta de alimento, espacio, etc.). Juntando estos dos momentos o principios se tiene básicamente la idea de selección natural. Dado que los individuos en una población son variables (no son

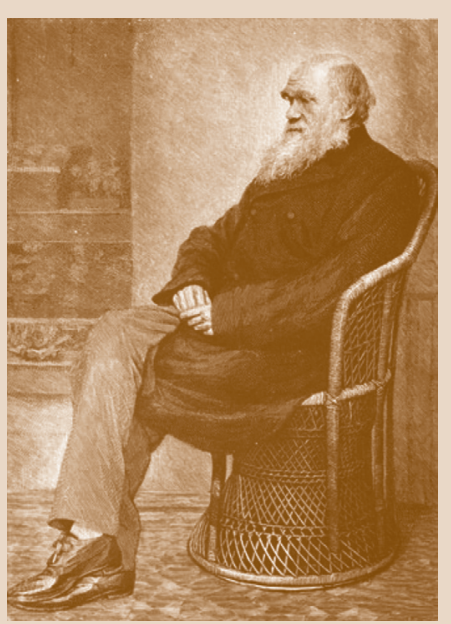

Ch. Darwin 
todos iguales) y que no todos pueden sobrevivir y dejar descendencia, dejarán un mayor número de descendientes aquellos individuos que porten alguna característica que les permita sobrevivir mejor que otros individuos de la misma población, según las condiciones del medio?.

Ahora bien, y siguiendo el hilo de nuestra exposición, debemos pensar esta idea de la selección natural y su relación con la técnica de su época. Decíamos antes que esta idea de selección tiene dos momentos: la variación poblacional azarosa y la competencia.

a) Empecemos por esta última idea y veamos desde dónde es pensada. Darwin dice expresamente que esta idea de competencia le ha venido de las ideas de Malthus. Para entender estas ideas, recordemos que desde fines del siglo XVIII se empieza a vivir la revolución industrial. El paulatino reemplazo de la mano de obra humana por la máquina conlleva a un aumento notable de la población y de la pobreza. Este desarrollo tecnológico "pone ante los ojos" el problema del recurso energético. No es de extrañar entonces que el siglo XIX sea el siglo de Marx, Darwin y la termodinámica. Esto es lo que vislumbra Malthus, quien no puede asumir las ideas de Lamarck sobre el progreso. Para Malthus, la inevitable falta de recursos hace que no toda la población humana pueda sobrevivir dado lo cual debemos esperar la pobreza y el hambre como consecuencias naturales de todo ello (Malthus, 1993, pp. 53-55). Es esta idea la que asume Darwin no sólo para el hombre, sino para todos los seres vivos. Darwin, por ello, tampoco cree en la evolución como una tendencia necesaria hacia el progreso ${ }^{10}$. Esta idea de competencia está, entonces, en natural consonancia con su época, en la que la mirada técnica ilumina las entidades en tanto que "recursos", los cuales comenzaban a escasear.

b) Por otra parte, atendamos ahora al principio de variación azarosa. Darwin lo entiende curiosamente como el "material" de la selección natu-

\footnotetext{
${ }^{9}$ Así lo explica Darwin: "Debido a esta lucha [por la vida] las variaciones, por ligeras que sean $[\ldots]$ si son en algún grado provechosas para los individuos de una especie [...] tenderán a la conservación de estos individuos y serán, en general, heredadas por la descendencia [...] He denominado a este principio, por el cual toda variación ligera, si es útil, se conserva, con el término de «selección natural», a fin de señalar su relación con la facultad de selección del hombre. Pero la expresión frecuentemente empleada por Mr. Herbert Spencer de la «supervivencia de los más aptos» es más exacta y, a veces, igualmente conveniente" (1936, p. 116).

${ }^{10}$ Así nos lo dice Darwin: "La selección natural, o la supervivencia de los más aptos, no implica necesariamente desarrollo progresivo, sólo saca provecho de las variaciones a medida que surgen y son beneficiosas para cada ser en sus complejas relaciones vitales" (1936, p. 180-181).
} 
$\mathrm{ral}^{11}$. Darwin concibe la selección natural, tal como lo dice la última cita, en clara analogía con la selección artificial que hace el hombre (domesticación). De hecho dedica todo el capítulo primero de su libro El origen de las especies a tal tema. La técnica humana ha intentado no sólo generar artefactos que imitan o completan la naturaleza como la sierra, o autómatas como el reloj, y no sólo controlar y manejar la técnica heredando este manejo a sus descendientes, sino que también ha intentado el control de los seres vivos que rodean al hombre para su utilidad. Para ello, disponiendo de las variantes ya existentes como "materiales" ha seleccionado aquellos organismos de interés haciendo que se reproduzcan en mayor medida que los que no le interesan ${ }^{12}$. La concepción de "material" de Darwin expuesta aquí no es otra que la ya pensada por Aristóteles desde la técnica griega. La variación poblacional azarosa es la fuente "potencial" desde donde puede haber selección. Ahora bien, es esta misma idea de "material" la que es traspasada a la teoría de selección natural de Darwin, entendiendo, claro está, que la naturaleza no es pensada aquí como un gigantesco sujeto que elija lo que debe ser conservado. El uso de este término es señalado expresamente por Darwin como metafórico. De ahí la complicación del término "selección natural" que en las últimas ediciones de su obra fue acompañado de otro término (proveniente de Spencer), no menos feliz, de "supervivencia del más apto". Pero, a pesar de que se indique que es un término metafórico, ello no puede ocultar que la selección natural es pensada desde la técnica humana de la domesticación. Esta concepción técnica muestra y destaca sólo una determinada faceta de los seres vivos que convendría seguir pensando: los muestra como una "sumatoria de cualidades" o, como hoy diríamos, de fenotipos. Estas cualidades se manifiestan cuando el hombre, apremiado por sus necesidades, pone atención a tales rasgos. El mismo Darwin se da cuenta de esto ${ }^{13}$. Aquí habría que meditar si tales cualidades fenotípicas "están ahí", son "naturales" y el hombre lo que hace es mera-

11 "Estas diferencias individuales son de la mayor importancia para nosotros, pues con frecuencia son heredables, como es sabido de todos, y proporcionan así materiales para que la selección natural actúe sobre ellas y las acumule, de la misma manera que el hombre acumula en una dirección determinada las diferencias individuales de sus producciones domésticas" (Darwin, 1983, p. 97, las cursivas son mías).

${ }^{12}$ Así, el hombre, en cierta manera, se ha ido generando, luego de muchas generaciones, a su "mejor amigo", el perro, eliminando, generación tras generación, a los perros más bravos.

13 "Pero, probablemente, el elemento más importante [para favorecer la selección] es que el animal o planta sea tan altamente estimado por el hombre que se preste la máxima atención aun a las desviaciones más leves de sus cualidades o estructuras. Sin poder prestar esa atención, no puede lograrse nada" (Darwin, 1983, p. 90). 
mente "atenderlas" o bien, en su afán de control, discretiza al organismo, lo vuelve una sumatoria de "partes". Más bien parece esto segundo ${ }^{14}$. En la selección artificial como técnica es claro que el organismo debe ser visto como compuesto de diversas "partes" fenotípicas, las cuales son seleccionadas para su beneficio. No es claro que ello ocurra en los seres vivos, que éstos estén compuestos de "partes" que se seleccionan ${ }^{15}$.

\section{CONCLUSIÓN}

Hemos intentado ver en este escrito, en cuatro grandes pensadores, la relación entre su pensamiento biológico y la técnica de su época. Aristóteles, veíamos, ha intentado comprender a los seres vivos a la "luz" de una determinada concepción de los artefactos griegos, como una casa o una sierra, los cuales han sido concebidos para imitar los entes naturales, como un cuchillo imita a unos dientes o una ropa a la piel. En este sentido, Aristóteles dice que "la técnica imita la naturaleza". Una casa, por ejemplo, se ha entendido como un conjunto de piedras que, de por sí, no están conectadas unas con otras, sino que se encuentran ordenadas a un fin ulterior como el constituir sus muros para dar abrigo. Este principio ordenador se lo da el técnico constructor de casas. Del mismo modo, se ha entendido las partes de un organismo como, por ejemplo, un diente, con la diferencia que su principio ordenador es intrínseco. De ahí entonces que si bien las propiedades "materiales" de un diente se explican por la "necesidad" de sus elementos componentes, su forma y función es explicada a la luz del organismo completo y, en definitiva, del cosmos. De este modo, Aristóteles ha tratado de entender a los seres vivos desde la técnica griega que fabrica objetos que imitan y completan a la naturaleza.

En segundo lugar, Descartes ha pensado a los seres vivos desde las máquinas “autómatas" como el reloj. Aquí las partes no están meramente sueltas las unas de las otras, sino que tienen cierta unidad intrínseca y una actividad "propia". El reloj tiene una organización intrínseca "superior" a

\footnotetext{
${ }^{14}$ Así lo cree Bertalanffy quien considera que la teoría de la selección natural fue pensada de forma "mecanicista" o analítica (1963, pp. 12-13).

${ }^{15}$ Conservando esta idea del organismo y sus "partes", Mendel, por ejemplo, ha fundado la genética en el supuesto de que hay fenotipos "discretos" como la semilla de arveja redonda o rugosa. Una mirada de esta semilla con el microscopio pondría esto en duda. A partir de tal concepción y de una serie brillante de experimentos se ha llegado a la idea de un gen discreto determinante de tal fenotipo discreto. Esto es algo que habría que meditar.
} 
la de una casa, aunque el reloj, en definitiva, debe su organización última al relojero. Siguiendo nuestro ejemplo biológico, dado más arriba, el diente sería ahora "visto" por Descartes como una parte de un engranaje funcional mecánico. En definitiva, Descartes intenta pensar los seres vivos desde los "objetos autómatas" que empezaban a proliferar en su época producto del pensamiento "analítico".

En tercer lugar, hemos visto el pensamiento de Lamarck. Él también piensa el organismo al modo mecánico con la diferencia, a mi juicio, de que atiende al momento de herencia cultural. Es como si viera, por ejemplo, el reloj no desde su mera individualidad, sino a la luz del "tiempo cultural". Aquí resalta la idea de progreso. El diente, siguiendo nuestro ejemplo, sería "visto" ahora como algo que, a partir del uso o desuso, se va haciendo, mejorando ciertas partes, si se usan, eliminándose otras, si no se usan, todo lo cual pasa a la descendencia. El diente iría "progresando" sucesivamente al modo de una plancha o un reloj. Lamarck ha resaltado de la técnica su evolución cultural y desde ahí ha meditado a los seres vivos en el tiempo.

En cuarto lugar, y finalmente, vimos el pensamiento de Darwin. Él piensa la selección natural como un proceso análogo al de la domesticación por parte del hombre, donde destacamos la variación poblacional azarosa como "material" o recurso, y la competencia que surge precisamente por la limitación energética intrínseca de la supervivencia de todas esas posibles variedades. El diente, por ejemplo, sería visto ahora surgiendo de un fondo material potencial de casi infinita variación y seleccionándose según las ventajas en la supervivencia y fecundidad que le conlleva al organismo que lo posee en función de su medio. Darwin ha resaltado de la técnica la selección artificial del hombre y desde ahí ha intentado iluminar a los seres vivos.

Dentro de esta línea investigada, podríamos intentar pensar qué sucede hoy. Al parecer, el ejemplo tecnológico por excelencia es el computador, con lo cual todo se reduce a "información". Habría que pensar si es esto mismo lo que orienta la investigación biológica actual. ¿Acaso no se reduce hoy todo a genes, entendidos justamente como unidades de información? ¿o se explicaría hoy el diente como algo determinado por una secuencia de información genética?

Para finalizar, me gustaría dejar planteadas algunas interrogantes. En estos autores se ha mostrado una cierta escisión o diferencia entre el mundo biológico entendido como "naturaleza" (como lo que es "en y por sí mismo", independiente del hombre) y la técnica como quehacer humano. En el estudio, hemos visto que los pensadores han usado como modelos 
ejemplos de la técnica y cultura humana, pero se nos diría que sólo son ejemplos analógicos o metafóricos. ¿Es esto verdad? ¿Es posible distinguir entre lo natural y lo técnico en el quehacer científico? ¿Acaso la investigación no dirige su mirada de acuerdo a los intereses y posibilidades de una época? ¿No es acaso el mundo técnico algo tan "familiar" al hombre que sólo desde aquí se puede intentar postular las explicaciones últimas de los seres vivos? Si esto fuera así, la técnica, tal vez, no sería un mero medio del hombre para producir cosas, sino una especie de horizonte intelectivo de posibilidades. Desde Zubiri, por ejemplo, la técnica podría ser meditada como un ámbito "campal" de intelección. Fue la idea de horizonte que animó a este escrito. Del mismo modo, Heidegger, como vimos, entendió la técnica, siguiendo a Aristóteles, como un "modo de verdad", de desvelación. Pero esta otra opción, llevada al extremo, deja a la ciencia, en cierto modo, como esclava de sus posibilidades culturales. ¿Es el horizonte de posibilidades culturales, por decirlo así, el que determina el curso de la ciencia? ¿Qué sucede con los experimentos, las nuevas experiencias registradas que la ciencia va aportando? ¿No aportan nada a la comprensión de los seres vivos? ¿No nos estaremos encandilando en la tiranía de la luz técnica? En fin, naturaleza y técnica son dos conceptos que debemos seguir meditando en su unidad y diferencia para tratar de comprender la gigantesca marcha del saber científico.

\section{REFERENCIAS}

Aristóteles (1995). Física (traducción de Guillermo R. de Echandía). Madrid: Gredos.

Aristóteles (2000). Metafísica (traducción de Tomás Calvo). Madrid: Gredos. Bertalanffy, L. (1963). Concepción biológica del cosmos. Santiago de Chile: Ediciones de la Universidad de Chile.

Darwin, C. (1983). El origen de las especies. Madrid: Sarpe.

Descartes, R. (1951). Los principios de la filosofía. Buenos Aires: Losada. . (2005). Las pasiones del alma. Madrid: Biblioteca Nueva.

Heidegger, M. (2001). "La pregunta por la técnica", en Conferencias y artículos (pp. 9-32). Barcelona: Del Serbal.

Lamarck, J. (1986). Filosofía zoológica. Barcelona: Alta Fulla.

Malthus, T. (1993). Primer ensayo sobre la población. Madrid: Alianza.

Mitcham, C. (1989). ¿Qué es la filosofía de la tecnología? Barcelona: Anthropos.

Ortega y Gasset, J. (2014). Ensimismamiento y alteración. Meditación de la técnica y otros ensayos. Madrid: Alianza.

Zubiri, X. (1982). Inteligencia y logos. Madrid: Alianza. . (2008). Sobre la esencia. Nueva edición. Madrid: Alianza. 